\title{
Comprehensive comparative outcomes in children with congenital heart disease: The rationale for the Congenital Catheterization Research Collaborative
}

\author{
Christopher J. Petit MD ${ }^{1}$ (D) | Athar M. Qureshi MD² | Andrew C. Glatz MD, MSCE ${ }^{3}$ (D) | \\ Courtney E. McCracken PhD ${ }^{1}$ | Michael Kelleman MSPH ${ }^{1}$ | George T. Nicholson MD ${ }^{4}$ | \\ Jeffery J. Meadows MD ${ }^{5}$ | Shabana Shahanavaz MBBS ${ }^{6}$ | Jeffrey D. Zampi MD ${ }^{7}$ | \\ Mark A. Law MD ${ }^{8}$ | Joelle A. Pettus MPH ${ }^{1}$ | Bryan H. Goldstein MD ${ }^{9}$ (D) \\ ${ }^{1}$ Sibley Heart Center Cardiology, Department of Pediatrics, Children's Healthcare of Atlanta, Emory University School of Medicine, Atlanta, Georgia \\ ${ }^{2}$ Lillie Frank Abercrombie Section of Cardiology, Department of Pediatrics, Texas Children's Hospital, Baylor College of Medicine, Houston, Texas \\ ${ }^{3}$ The Cardiac Center, Children's Hospital of Philadelphia, Department of Pediatrics, Perelman School of Medicine, University of Pennsylvania, Philadelphia, \\ Pennsylvania \\ ${ }^{4}$ Division of Cardiology, Department of Pediatrics, Vanderbilt University School of Medicine, Nashville, Tennessee \\ ${ }^{5}$ Division of Cardiology, Department of Pediatrics, University of California San Francisco School of Medicine, San Francisco, California \\ ${ }^{6}$ Section of Pediatric Cardiology, Department of Pediatrics, Washington University School of Medicine, St Louis, Missouri \\ ${ }^{7}$ Division of Cardiology, Department of Pediatrics, CS Mott Children's Hospital, University of Michigan School of Medicine, Ann Arbor, Michigan \\ ${ }^{8}$ Division of Pediatric Cardiology, Department of Pediatrics, Children's of Alabama, University of Alabama Birmingham School of Medicine, Birmingham, \\ Alabama \\ ${ }^{9}$ The Heart Institute, Cincinnati Children's Hospital Medical Center, Department of Pediatrics, University of Cincinnati College of Medicine, Cincinnati, Ohio
}

\section{Correspondence}

Christopher J. Petit, MD, Department of Pediatrics, Emory University School of Medicine, 1405 Clifton Rd NE, Atlanta, GA 30322

Email: cjpetit@emory.edu

\section{Abstract}

Clinical research in the treatment of patients with congenital heart disease (CHD) is limited by the wide variety of CHD manifestations and therapeutic options as well as the generally low incidence of CHD. The availability of comprehensive, contemporary outcomes studies is therefore limited. This inadequacy may result in a lack of data-driven medical decision making. In 2013, clinician scientists at two centers began a research collaboration, the Congenital Catheterization Research Collaborative (CCRC). Over time, the CCRC has grown to include nine cardiac centers from across the United States, with a common data coordinating center. The CCRC seeks to generate high-quality, contemporary, statistically robust, and generalizable outcomes research which can help address important clinical questions in the treatment of CHD. To date, the CCRC has reported on multicenter outcomes in: neonates with congenital aortic stenosis, infants undergoing right ventricular decompression for pulmonary atresia and intact ventricular septum, and infants with ductal-dependent pulmonary blood flow. The CCRC has been successful at leveraging large multicenter cohorts of patients in a contemporary period to perform comparative studies. In the future, the CCRC plans to continue to perform hypothesis-driven retrospective and prospective observational studies of CHD populations where controversy exists or 
where novel interventions or therapies have emerged. Quality improvement efforts including lesion-specific registry development may be an additional potential future target.

\section{KEYWORDS}

Collaboration, Multicenter, Research

\section{1 | INTRODUCTION}

A high degree of variability attends nearly all forms of therapy for patients with congenital heart disease (CHD). This variability is inherent in medicine and reflects a number of immovable features of health care delivery. Temporal variability is likely due to factors such as improved understanding of pathophysiology and improved therapeutics. Technological advances have not only continued to emerge, but have been introduced at an accelerating rate over the past 3 decades. In areas as disparate as ventricular assist devices, stent technology, and anticoagulation therapies, innovations are introduced regularly. Regional and center-based variability reflects geographic and at times individual practitioner preferences, but may also reflect population and cultural biases, preconceptions, and vulnerabilities.

These variations make it difficult to interpret outcomes following both established, conventional therapies as well as novel interventions. The difficulty is particularly notable in the field of pediatric cardiology, where small populations with anatomically heterogenous cardiac malformations seemingly preclude comprehensive review and statistical comparison. For example, despite the fact that patent ductus arteriosus (PDA) stenting has been performed in neonates with cyanotic CHD since 1991, the largest single-center outcome studies evaluating the safety and efficacy of this procedure have been limited to small cohorts of 8-64 patients, with larger cohorts spanning broad eras (Figure 1). ${ }^{1-8}$ Over the inclusion periods in those studies, concomitant advances in catheter and stent technology make it difficult to generalize the published outcomes reflecting older technologies to contemporary practice. Further, with no appropriate control arms in such studies, determination of superiority of therapy is not possible.

Given the relatively low incidence of particular forms of CHD, and institutional and regional practice patterns, we sought to use a multicenter approach to CHD research to mitigate these hindrances. We created a multicenter research collaborative, now termed the Congenital Catheterization Research Collaborative (CCRC), in 2013. Originally consisting of two centers, the CCRC is now comprised of nine pediatric cardiac centers from across the United States. By design, the CCRC includes both medium- and high-volume geographically diverse cardiac centers. Despite differences in treatment strategies in some areas of CHD, all centers offer comprehensive cardiac care, and importantly have complementary clinical and academic missions. The goal of the CCRC is to enhance the understanding of the pathophysiology of CHD and the outcomes following CHD interventions. Our research methods rely on leveraging multicenter,

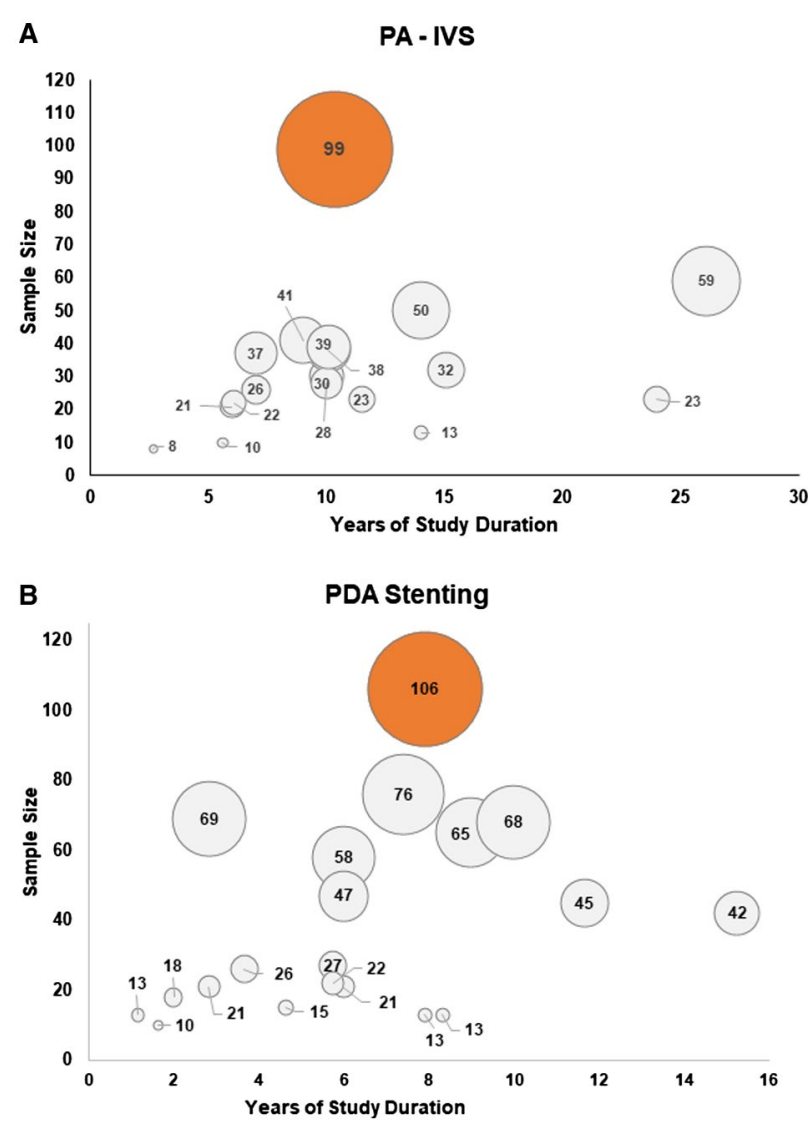

FIGURE 1 Pediatric cardiology studies compared by span of years and size of Cohort included. These scatter plots demonstrate the range of studies reporting outcomes on pulmonary atresia with intact ventricular septum (PA-IVS) in Panel A, and on stenting of the patent ductus arteriosus (PDA) in Panel B. Size of cohort is indicated on the vertical axes, and span of study (years) is indicated on the horizontal axes. Previous publications in PA-IVS spanned many years, up to 28 years, in order to achieve cohorts of 40-60 patients. Conversely, the CCRC PA-IVS study included 99 patients in a span of 10 years (orange circle) (A). Studies evaluating outcomes following PDA stenting (B) likewise included small cohorts or conversely achieved larger cohorts by including procedures performed $>10$ years earlier. However, the CCRC PDA stent study included 106 neonates in an 8-year span (orange circle)

contemporary clinical data from our network of diverse cardiac centers.

The mission of the CCRC is to improve the outcomes for patients with CHD by undertaking hypothesis-driven research with a special 
focus on areas of innovation within pediatric and adult congenital interventional cardiology. The goal of the current paper is to describe the rationale for the creation of the CCRC, the projects achieved to date, and the future directions of our collaboration.

\section{2 | HISTORY}

The CCRC began in 2013 when clinician scientists from Cincinnati Children's Hospital Medical Center and Children's Healthcare of Atlanta collaborated to study the relationship between valve morphology and outcomes following balloon aortic valvuloplasty. ${ }^{9}$ The collaboration proved successful not only academically, but because the collaborating investigators found they could reliably work together, across geographic and technological barriers. Web-based video conference calls were held where echocardiograms and catheterization angiograms were jointly reviewed and interpreted. These video conferences facilitated, for example, consistent measurements of unique, nuanced echocardiographic parameters. This allowed for a high degree of interobserver reliability even in the setting of challenging anatomic substrates. The multicenter approach led to enhanced appreciation for differing approaches to aspects of patient care with congenital aortic stenosis, including methods of valve annulus measurement and technical performance of balloon aortic valvuloplasty.

In 2015, the CCRC grew to include investigators from the Children's Hospital of Philadelphia and Texas Children's Hospital. The resultant larger CCRC group, which would go on to form the executive committee, sought to understand factors associated with poor outcomes in children with pulmonary atresia and intact ventricular septum (PA-IVS). Previous reports on PA-IVS had largely been limited to single-center, small cohort studies, and many of the larger series were hindered by a broad span of inclusion dates (some spanning multiple decades of institutional experience), or very small cohorts where more contemporary approaches were employed but statistical power was wanting (Figure 1). ${ }^{10-16}$ The CCRC studied a contemporary, relatively large cohort of neonates with PA-IVS. We evaluated a host of echocardiographic and hemodynamic factors and identified that preintervention tricuspid regurgitation was associated with a host of important clinical end points following right ventricle decompression in neonates with PA-IVS. ${ }^{17}$ Additional analyses led to an enhanced understanding of the importance of nuanced technical variables on outcomes. As an example, we reported that higher radiofrequency energy application was associated with complications such as cardiac perforation. ${ }^{18}$

The study which the CCRC pursued next was a comparison of outcomes following transcatheter PDA stenting versus surgical systemic-to-pulmonary artery shunts (ie, BT shunts) in infants with ductal-dependent pulmonary blood flow. Prior to this study, published reports were limited to small case series of PDA stenting procedures or rarely, even smaller cohorts where outcomes following PDA stenting were compared to those following BT shunt. In both types of studies, generalization of results was restricted by small cohort size, poor statistical power, and institutional preferences. Particularly in the case of the comparative studies, results were confounded by indication, as neonates undergoing PDA stenting had undeniably different risk factors when compared to the BT shunt cohorts. ${ }^{3,8}$ The CCRC leveraged a relatively large multicenter population, all palliated within the past 10 years, which reflects the current era of surgical and transcatheter techniques and available devices. Importantly, this cohort size allowed for propensity score adjustment to account for confounding by indication, including inherent differences in cardiac anatomy, expected physiology, and other patient- and center-specific factors. ${ }^{19}$ Ensuing studies from this cohort were performed which again highlighted important technical factors to consider when performing PDA stenting, the impact of the underlying PDA morphology on the outcomes following PDA stenting, and the impact of palliative strategy on the cost of care.

Investigators from five additional institutions (Table 1) have since joined the CCRC including members from Vanderbilt University, University of Alabama-Birmingham, University of California San Francisco, University of Michigan, and Washington University in St. Louis. New and ongoing studies, outlined below, will include patients treated at all 9 centers. With the inclusion of new members to the CCRC, the executive committee recognized a need to both formalize the processes for conducting research and also to introduce the CCRC methodology to new investigators. For this reason, we developed an onboarding structure for new members which helps each investigator to understand the roles, expectations, and mechanisms the site principal investigator assumes within the collaborative.

Whenever possible, the CCRC draws from expertise beyond the membership of the CCRC. Cardiology subspecialists in noninvasive imaging, in particular, are important collaborators and indeed leaders of research efforts. ${ }^{20}$ In these studies, imaging specialists have played an important role in assigning anatomic risk factors. ${ }^{9,17}$ Surgical colleagues were included from the outset in our first comparative study between BT shunt and PDA stent. ${ }^{19}$ The CCRC also places a high priority on mentoring of junior faculty and trainees, with active roles in research projects. Close collegial relationships with other important members of the congenital cardiac community help ensure our work minimizes potential bias and reflects the best available science.

\section{3 | CCRC CULTURE}

While developing our bylaws and guidelines, the CCRC executive committee conferred with representatives from established and respected collaborative organizations, in particular leaders from the Pediatric Heart Network (PHN), the Pediatric Heart Transplant Study (PHTS), the Pediatric Cardiac Critical Care Collaborative $\left(\mathrm{PC}^{4}\right)$, and the Pediatric Acute Care Cardiology Collaborative (PAC3). ${ }^{21-24}$ These collaborations each have unique goals, administrative structures, and data collection instruments and yet their experiences and guidance were invaluable in creating the administrative and academic 
TABLE 1 Members of the congenital catheterization research collaborative

\begin{tabular}{|c|c|c|c|}
\hline Individual & University affiliate & Hospital affiliate & Membership \\
\hline Christopher J. Petit, MD & $\begin{array}{l}\text { Emory University School of Medicine, } \\
\text { Atlanta, Georgia }\end{array}$ & Children's Healthcare of Atlanta & $\begin{array}{l}\text { President } \\
\text { Member since } 2013\end{array}$ \\
\hline Bryan H. Goldstein, MD & University of Cincinnati, Cincinnati, Ohio & $\begin{array}{l}\text { Cincinnati Children's Hospital } \\
\text { Medical Center }\end{array}$ & $\begin{array}{l}\text { Vice-President } \\
\text { Chair, Finance Committee } \\
\text { Member since } 2013\end{array}$ \\
\hline Courtney M. McCracken, PhD & $\begin{array}{l}\text { Emory University School of Medicine, } \\
\text { Atlanta, Georgia }\end{array}$ & Children's Healthcare of Atlanta & $\begin{array}{l}\text { Chair-Biostatistics } \\
\text { Member since } 2013\end{array}$ \\
\hline Athar M. Qureshi, MD & Baylor College of Medicine, Houston, Texas & Texas Children's Hospital & $\begin{array}{l}\text { Chair-New Studies } \\
\text { Committee } \\
\text { Member since } 2015\end{array}$ \\
\hline Andrew C. Glatz, MD MSCE & $\begin{array}{l}\text { University of Pennsylvania, Philadelphia, } \\
\text { Pennsylvania }\end{array}$ & Children's Hospital of Philadelphia & $\begin{array}{l}\text { Chair-Scientific } \\
\text { Committee } \\
\text { Member since } 2015\end{array}$ \\
\hline George T. Nicholson, MD & Vanderbilt University, Nashville, Tennessee & $\begin{array}{l}\text { Monroe Carell Jr Children's } \\
\text { Hospital }\end{array}$ & Member since 2017 \\
\hline Jeffery Meadows, MD & $\begin{array}{l}\text { University of California-San Francisco, San } \\
\text { Francisco, California }\end{array}$ & UCSF Benioff Children's Hospital & Member since 2017 \\
\hline Jeffrey D. Zampi, MD & University of Michigan, Ann Arbor, Michigan & CS Mott Children's Hospital & Member since 2018 \\
\hline Shabana Shahanavaz, MBBS & Washington University, St. Louis, Missouri & St. Louis Children's Hospital & Member since 2018 \\
\hline Mark A. Law, MD & $\begin{array}{l}\text { University of Alabama-Birmingham, } \\
\text { Birmingham, Alabama }\end{array}$ & Children's of Alabama & Member since 2018 \\
\hline Joelle A. Pettus, MPH MSW & $\begin{array}{l}\text { Emory University School of Medicine, } \\
\text { Atlanta, Georgia }\end{array}$ & Children's Healthcare of Atlanta & $\begin{array}{l}\text { Program Manager since } \\
2018\end{array}$ \\
\hline
\end{tabular}

structure of our collaboration. Leaders from these collaborations are routinely invited to CCRC's in-person meetings to provide ongoing advice to the executive committee. Similarly, members of the CCRC have been invited to serve on local and national career development panels-particularly focusing on the benefits academically and professionally associated with multicenter collaborative research.

Several attributes separate the CCRC from other seemingly similar groups. One important distinction is that the CCRC is not a quality improvement registry such as the Improving Pediatric and Adult Congenital Treatments (IMPACT) Registry, Congenital Cardiac Catheterization Project on Outcomes (C3PO), or PC4. ${ }^{23,25,26}$ Rather, the CCRC undertakes hypothesis-driven retrospective and prospective observational research, the focus of which is often, although not exclusively, related to outcomes following interventional procedures.

Given the inherent limitations of retrospective research, it is imperative that the CCRC develops and adheres to rigorous methods and timely data collection to enhance the rigor, quality, and value of our research. These critical methods include:

A-use of contemporary clinical data reflecting up-todate interventional, imaging, and surgical approaches.

B-highly granular and accurate data collection reflecting a comprehensive data auditing process.
C-pooling of contemporary data across multiple sites, providing increased statistical power despite relatively short inclusion periods.

Each study proposal undergoes a review by the New Study Proposal Committee chair as well as the Executive Committee. Proposals with well-defined clinical end points and compelling clinical questions are prioritized. The CCRC reviews such proposals and has adopted a scoring format based upon the National Institutes of Health Study Section process. ${ }^{27}$ After the review of proposals is undertaken, necessary data elements are provided and a case report form (CRF), data dictionary, and Manual of Operations (MOO) are created. Data programming within Research Electronic Data Capture (REDCap) allows for secure web-based electronic data entry among the CCRC sites. ${ }^{28}$ The databases are created and maintained by the CCRC data coordinating center (DCC), The Children's Healthcare of Atlanta.

Another critical aspect of the CCRC is our culture of transparency and democratic structure. Specifically, the expectation among the CCRC's participants is that all investigators share in the academic workload, the academic credit as well as the organizational responsibilities. Authorship responsibilities and assignments rotate so that each member of the CCRC remains fully engaged, enjoys professional growth and satisfaction, and can ultimately help mentor future CCRC members. This concept, we believe, will enable the CCRC to remain a high-quality clinically relevant research collaboration for 
years to come, as more junior members begin to lead CCRC's academic and organizational efforts.

\section{4 | CCRC ORGANIZATIONAL STRUCTURE AND FINANCES}

The CCRC has developed a number of committees, each of which focuses on a unique aspect of our mission (Figure 2). The executive committee comprised of 4 founding members of the CCRC plus 1-2 at-large members who are elected to serve 2 -year terms. The role of the executive committee is to ensure the goals and mission of the CCRC are sustained, that academic roles and credit are appropriately and equitably distributed, and that studies continue to be completed efficiently but also with appropriate scientific rigor. Committees function to enhance the ability of the CCRC to undertake both large-scale and focused projects. The Finance Chair oversees the fiscal budget, oversees payment of dues, and assists with philanthropic efforts. The Scientific Committee Chair advises each PI during the formulation of each new study and oversees the analytic plan. The Biostatistics Chair advises investigators on methodological approaches which enhance the capabilities to perform rigorous statistics, to minimize selection bias, and to control for other obvious and subtle confounders. The New Studies Chair focuses on evaluating new study proposals not only to evaluate scientific merit, but also to prioritize studies, and to anticipate and promote affiliated, ancillary studies.

Finances of the CCRC are sustained through a combination of annual member dues as well as philanthropy. Each principal investigator's institution has committed to annual dues which support, to date, the majority of the administrative functions of the CCRC. The costs associated with biannual in-person meetings are largely underwritten by the CCRC. Currently, the CCRC enjoys important philanthropic support from generous donors who support the mission of the CCRC as well as the individual clinicians who comprise the collaborative. In many cases, these donors are parents of children or relatives with congenital heart disease. To date, philanthropic support has facilitated the hiring of a program manager, who is based at the DCC and oversees the regulatory and administrative functions of the CCRC. Grant funding sources will be considered as the collaborative focus on larger scale prospective observational studies.

The CCRC holds a biannual in-person meeting in Atlanta, at the DCC. These 2-3-day in-person meetings are critical for both academic success and organizational momentum. The meetings allow for in-depth academic project review, including evaluation of study analyses with investigators and statisticians on site, with presentations and updates given by the principal investigator of each unique study and writing group. Further, the meetings allow for new study proposals to be presented, refined, and planned. The CCRC members also use the meetings to discuss the organizational strategic vision, review CCRC finances, and plan for the introduction of new members when appropriate. The in-person meetings, combined with monthly conference calls and webinars, ensure regular communication and an open culture where ideas, criticisms, and disagreements are shared, and a durable collegial relationship among members is both established and maintained. Importantly, the professional and social network which has developed within the collaborative over time among the CCRC investigators has facilitated an ongoing dedication to career development, professional support, and leadership development for all CCRC members.

\section{5 | CCRC DATA QUALITY AND AUDITING}

The data auditing process has matured over the initial years of the CCRC's existence. With larger cohort studies involving an increasingly broad span of data points, it became necessary to introduce

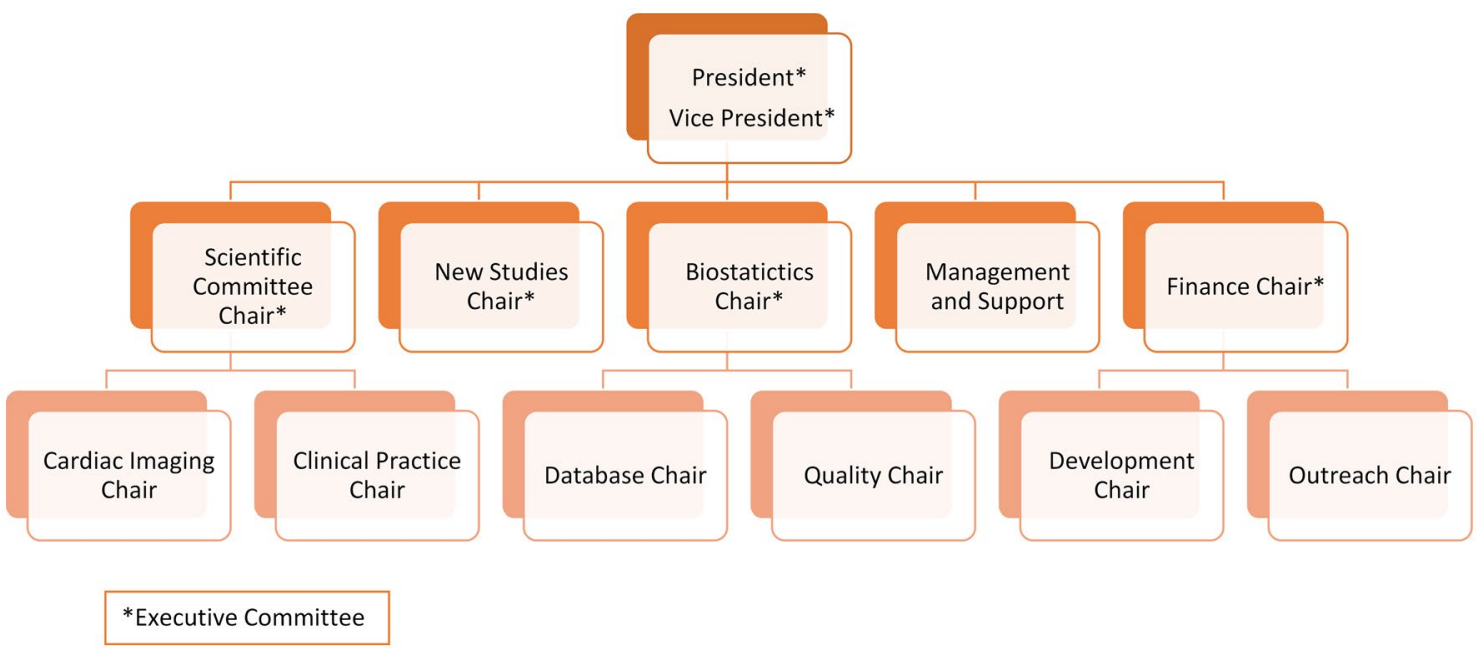

FIGURE 2 Congenital catheterization research collaborative administrative structure. This graph details the leadership and the administrative structure of the Congenital Catheterization Research Collaborative (CCRC). Roles within the CCRC are distributed and may rotate to ensure that each member is highly engaged in the administrative and academic functions of our collaborative 
a thorough auditing process. The executive committee, again, discussed the issue of data quality with leaders of other multicenter collaboratives, notably the $\mathrm{PC}^{4}$ registry. Ultimately, we utilize a 2step approach to ensure data quality: data entry training and interval data auditing.

Prior to the opening of new study REDCap databases, the CCRC Program Manager and Biostatistics Chair develop a study-specific training module for all CCRC principal investigators, study coordinators, and affiliated data entry personnel from each site. REDCap data entry is reviewed, with Data Dictionary elements and the MOO explained. Each site enters 2-3 sample patients and then these are reviewed with the CCRC Program Manager. Once the entries are reviewed and the data entry personnel from a given site understand the data elements and nature of data entry, the site is then permitted to proceed with complete data entry for that study.
The program manager, study biostatisticians as well as the study lead investigator with assistance from the Scientific Committee chair will conduct audits of data on regular intervals. Random cases (10\%-20\% of total cohort per site), assigned by the Biostatistics Chair, will be reviewed by: (1) notification of the site principal investigator so that primary documents (eg, operative reports, echocardiogram reports, catheterization reports) can be electronically captured and uploaded into REDCap and (2) primary document review at the DCC. Auditors will then adjudicate the entered data with the primary source documents to ensure completeness and accuracy of data entry. Discrepancies will then be resolved. When systematic, recurring errors are noted, retraining of the involved site personnel may be necessary. Errors in data extraction and entry are recorded and these challenges in data entry discussed during monthly conference calls to ensure maximal efficiency and mutual benefit across all sites.

TAB LE 2 List of congenital catheterization research collaborative publications and studies to date

Relation of aortic valve morphologic characteristics to aortic valve insufficiency and residual stenosis in children with congenital aortic stenosis undergoing balloon valvuloplasty ${ }^{30}$

Aortic valve morphology correlates with left ventricular systolic function and outcome in children with congenital aortic stenosis prior to balloon aortic valvuloplasty ${ }^{9}$

Outcomes after decompression of the right ventricle in infants with pulmonary atresia with intact ventricular septum are associated with degree of tricuspid regurgitation: results from the Congenital Catheterization Research Collaborative ${ }^{17}$

A comparison between patent ductus arteriosus stent and modified Blalock-Taussig shunt as palliation for infants with ductal-dependent pulmonary blood flow: insights from the congenital catheterization research collaborative ${ }^{31}$

Technical factors are associated with complications and repeat intervention in neonates undergoing transcatheter right ventricular decompression for pulmonary atresia and intact ventricular septum: results from the Congenital Catheterization Research Collaborative ${ }^{32}$

Echocardiographic predictors of biventricular physiology in pulmonary atresia and intact ventricular septum: results from the congenital catheterization research collaborative ${ }^{20}$

Classification scheme for ductal morphology in cyanotic patients with ductal-dependent pulmonary blood flow and association with outcomes of patent ductus arteriosus stenting ${ }^{33}$

Blalock-Taussig shunt versus patent ductus arteriosus stent as first palliation for ductal dependent pulmonary circulation lesions: a review of the literature ${ }^{34}$

Stenting of the ductus arteriosus for ductal-dependent pulmonary blood flow-current techniques and procedural considerations ${ }^{35}$

Use of carotid and axillary artery approach in patent ductus arteriosus stenting: a multicenter study from the congenital catheterization research collaborative ${ }^{36}$

Differences in cost by palliation strategy for infants with ductal-dependent pulmonary blood flow: analysis of data from the congenital catheterization research collaborative ${ }^{37}$

Impact of treatment strategy on outcomes in isolated pulmonary artery of ductal origin: a multicenter report from the congenital catheterization research collaborative ${ }^{38}$

Comparison of ductal arteriosus stent and Blalock-Taussig shunt as palliation for neonates with sole source ductal-dependent pulmonary blood flow: results from the congenital catheterization research collaborative ${ }^{39}$

Differences in somatic growth and mode of feeding by palliation strategy for infants with ductal-dependent pulmonary blood flow: results from the congenital catheterization research collaborative $^{18}$

Comparison of outcomes at time of superior cavopulmonary connection between single ventricle patients with ductal-dependent pulmonary blood flow initially palliated with either Blalock-Taussig shunt or ductus arteriosus stent: results from the Congenital Catheterization Research Collaborative ${ }^{19}$
American Journal of Cardiology-2016

Journal of Invasive Cardiology-2016

Circulation: Cardiovascular

Interventions-2017

Circulation-2018

Cardiology in the Young-2018

Congenital Heart Disease-2018

Catheterization and Cardiovascular Interventions-In Press

Congenital Heart Disease-2018

Congenital Heart Disease-2018

Under review

Under review

Abstract Presentation at Society for Cardiovascular Angiography and Interventions 2018

Abstract Presentation at American Heart Association 2018

Abstract Presentation at American Heart Association 2018

Abstract Presentation at American Heart Association 2018 
Whenever possible, data extraction for specific studies will be enhanced by utilizing site-specific data warehouses-eg, Lumedx (Cardiovascular Data Intelligence, Oakland, California) or CardioAccess (CardioAccess Inc, Fort Lauderdale, Florida)-which offer the benefit of prior review and confirmation of all datapoints by each site's internal bioinformatics team. Use of institutional data warehouses is achieved using common diagnostic or procedural codes derived from either the Improving Pediatric and Adult Congenital Treatment (IMPACT) registry or the Society of Thoracic Surgeons (STS) Congenital database. ${ }^{26,29}$ Use of these common codes across the registries (and therefore across CCRC centers, all of which participate in these registries) ensures standardization, appropriate patient inclusion, and common procedure definitions. The CCRC has also linked patient identifiers at the center-level to Pediatric Health Information System (PHIS) data to perform health care cost comparison analysis. These finance data are then deidentified and reviewed and analyzed in a blinded manner.

\section{6 | CCRC FUTURE DIRECTIONS}

To date, the CCRC has undertaken strictly retrospective, comprehensive outcomes studies (Table 2). Our current study will compare midterm and late outcomes in symptomatic (cyanotic) neonates with tetralogy of Fallot (TOF), based upon initial treatment strategy. The 2 main cohorts will be those undergoing primary neonatal TOF repair and those neonates undergoing palliation (including BT shunt, PDA stent, right ventricular outflow tract stent, and balloon pulmonary valvuloplasty) with subsequent complete TOF repair. We anticipate that after the creation of this large multicenter database, multiple derivative studies will be undertaken to answer more focused clinical questions.

We also aim to undertake prospective, observational studies involving populations with $\mathrm{CHD}$-including cohorts previously studied in a retrospective manner. Future potential studies include the evaluation of neurodevelopmental outcomes following catheter-based palliation for infants with CHD, comparative studies based on intention-to-treat analysis (which is unachievable in a retrospective study), and observational cohort studies of high-risk CHD lesions. For these prospective studies, we anticipate considerably increased work burden at each site, which will require additional coordinator support to facilitate sustained success. In an effort to mitigate this added effort, we plan to develop center-level data linkages to existing registries, including PC4, PAC3, STS, and IMPACT, to reduce the work burden of chart abstraction.

Lastly, we recognize the increasing role-and at times overlap-of discipline-specific large multicenter collaboratives within the field of pediatric and adult congenital cardiology. In order to offer value not just to investigator members, but to the hospitals and academic institutions which financially sustain these organizations, many of these collaboratives operate within the sphere of quality improvement work. Quality improvement initiatives further institutional missions, and therefore registry-based multicenter efforts are perceived as directly applicable in this light. With the development of prospective research projects, as detailed above, we intend for the CCRC to offer similar value, with access to continuous feedback on patient outcomes as well as highly technical procedural details, in part to facilitate the introduction of novel treatment strategies. Lesion-specific registry development may also be a focus of future CCRC work to facilitate discrete quality improvement efforts related to interventional procedures and their outcomes. We also plan to study patient-reported outcomes in future studies, taking advantage of the existing and ensuing identified populations. We anticipate that the CCRC will continue to evolve, from a retrospective-based research collaborative into a multicenter prospective research group focusing on improving outcomes, establishing procedural benchmarks, and performing comparative research. We believe that hypothesis-driven research is a critical tool for quality improvement, and data-driven clinical decision making leads to optimized care of patients with CHD.

\section{7 | CONCLUSION}

The CCRC is a multicenter research collaborative which aims to improve the health and well-being of patients with CHD. Our approach of utilizing highly granular clinical data allows us to evaluate patientspecific and procedural factors and their effect on outcomes following interventions. Our goal is to provide scientific data to optimize clinical decision making, particularly where controversy or disagreement exists. We believe that the CCRC, through partnerships with existing cardiac registries and with our institutional colleagues, will help improve our understanding of the outcomes following CHD interventions.

\section{ACKNOWLEDGMENTS}

We would like to thank David Naftel PhD of the University of Alabama-Birmingham, Sara Pasquali MD of the University of Michigan, and William Mahle MD of Emory University for their assistance, suggestions, and support, particularly in the early stages of the CCRC establishment. The CCRC is also indebted to the Kennedy Hammill Research Foundation for financial support.

\section{CONFLICT OF INTEREST}

None.

\section{AUTHOR CONTRIBUTIONS}

Conceived, drafted, edited the manuscript: Christopher J. Petit Involved in initial conception, drafting and editing of manuscript: Athar M. Qureshi, Andrew C. Glatz, Courtney E. McCracken, Michael Kelleman, George T. Nicholson, Jeffery J. Meadows, Shabana Shahanavaz, Jeffrey D. Zampi, Mark A. Law, Bryan H. Goldstein

Involved in creating graphics, drafting and editing of manuscript: Joelle A. Pettus 


\section{ORCID}

Christopher J. Petit iD http://orcid.org/0000-0002-9939-3048

Andrew C. Glatz (iD) http://orcid.org/0000-0003-3791-8280

Bryan H. Goldstein (iD http://orcid.org/0000-0001-8508-9523

\section{REFERENCES}

1. Gibbs JL, Rothman MT, Rees MR, Parsons JM, Blackburn ME, Ruiz CE. Stenting of the arterial duct: a new approach to palliation for pulmonary atresia. Br Heart J. 1992;67:240-245.

2. Alwi M, Choo KK, Latiff HA, Kandavello G, Samion H, Mulyadi MD. Initial results and medium-term follow-up of stent implantation of patent ductus arteriosus in duct-dependent pulmonary circulation. J Am Coll Cardiol. 2004;44:438-445.

3. Amoozgar H, Cheriki S, Borzoee M, et al. Short-term result of ductus arteriosus stent implantation compared with surgically created shunts. Pediatr Cardiol. 2012;33:1288-1294.

4. Santoro G, Gaio G, Giugno L, et al. Ten-years, single-center experience with arterial duct stenting in duct-dependent pulmonary circulation: early results, learning-curve changes, and mid-term outcome. Catheter Cardiovasc Interv. 2015;86:249-257.

5. Sivakumar K, Bhagyavathy A, Coelho R, Satish R, Krishnan P. Longevity of neonatal ductal stenting for congenital heart diseases with duct-dependent pulmonary circulation. Congenit Heart Dis. 2012;7:526-533.

6. Michel-Behnke I, Akintuerk H, Thul J, Bauer J, Hagel KJ, Schranz D. Stent implantation in the ductus arteriosus for pulmonary blood supply in congenital heart disease. Catheter Cardiovasc Interv. 2004;61:242-252.

7. Gewillig M, Boshoff DE, Dens J, Mertens L, Benson LN. Stenting the neonatal arterial duct in duct-dependent pulmonary circulation: new techniques, better results. J Am Coll Cardiol. 2004;43:107-112.

8. Mallula K, Vaughn G, El-Said H, Lamberti JJ, Moore JW. Comparison of ductal stenting versus surgical shunts for palliation of patients with pulmonary atresia and intact ventricular septum. Catheter Cardiovasc Interv. 2015;85:1196-1202.

9. Gao K, Sachdeva R, Goldstein BH, Lang S, Petit CJ. Aortic valve morphology correlates with left ventricular systolic function and outcome in children with congenital aortic stenosis prior to balloon aortic valvuloplasty. J Invasive Cardiol. 2016;28:381-388.

10. Chubb H, Pesonen E, Sivasubramanian S, et al. Long-term outcome following catheter valvotomy for pulmonary atresia with intact ventricular septum. J Am Coll Cardiol. 2012;59:1468-1476.

11. Schwartz MC, Glatz AC, Dori Y, Rome JJ, Gillespie MJ. Outcomes and predictors of reintervention in patients with pulmonary atresia and intact ventricular septum treated with radiofrequency perforation and balloon pulmonary valvuloplasty. Pediatr Cardiol. 2014;35:22-29.

12. Hasan BS, Bautista-Hernandez V, McElhinney DB, et al. Outcomes of transcatheter approach for initial treatment of pulmonary atresia with intact ventricular septum. Catheter Cardiovasc Interv. 2013;81:111-118.

13. Justo RN, Nykanen DG, Williams WG, Freedom RM, Benson LN. Transcatheter perforation of the right ventricular outflow tract as initial therapy for pulmonary valve atresia and intact ventricular septum in the newborn. Cathet Cardiovasc Diagn. 1997;40:408-413.

14. Alwi M, Geetha K, Bilkis AA, et al. Pulmonary atresia with intact ventricular septum percutaneous radiofrequency-assisted valvotomy and balloon dilation versus surgical valvotomy and Blalock Taussig shunt. J Am Coll Cardiol. 2000;35:468-476.

15. Hanley FL, Sade RM, Blackstone EH, Kirklin JW, Freedom RM, Nanda NC. Outcomes in neonatal pulmonary atresia with intact ventricular septum. A multiinstitutional study. J Thorac Cardiovasc Surg. 1993;105:406-423.

16. Ashburn DA, Blackstone EH, Wells WJ, et al. Determinants of mortality and type of repair in neonates with pulmonary atresia and intact ventricular septum. J Thorac Cardiovasc Surg. 2004;127(4):1000-1008.

17. Petit CJ, Glatz AC, Qureshi AM, et al. Outcomes after decompression of the right ventricle in infants with pulmonary atresia with intact ventricular septum are associated with degree of tricuspid regurgitation: results from the congenital catheterization research collaborative. Circ Cardiovasc Interv. 2017;10:pii: e004428.

18. Nicholson G, Glatz AC, Qureshi A, et al. Differences in somatic growth and feeding regimen by palliation strategy for infants with ductal-dependent pulmonary blood flow: results from the Congenital Catheterization Research Collaborative. Circulation. 2018;138:A15310.

19. Meadows J, Qureshi A, Goldstein B, et al. Comparison of outcomes at time of superior cavopulmonary bonnection between single ventricle patients with ductal-dependent pulmonary blood flow Initially palliated with either Blalock-Taussig shunt or ductus arteriosus stent: results from the Congenital Catheterization Research Collaborative. Circulation. 2018;138:A16167.

20. Maskatia SA, Petit CJ, Goldstein BH, et al. Echocardiographic parameters associated with clinical outcome and right ventricular growth following right ventricular decompression in patients with pulmonary atresia intact ventricular septum: results from the congenital catheterization research collaborative. Congenit Heart Dis. 2018. Sep 21 [Epub ahead of print].

21. Mahony L, Sleeper LA, Anderson PA, et al. GD and pediatric heart network I. The pediatric heart network: a primer for the conduct of multicenter studies in children with congenital and acquired heart disease. Pediatr Cardiol. 2006;27:191-198.

22. Canter C, Naftel D, Caldwell R, et al. Survival and risk factors for death after cardiac transplantation in infants. A multi-institutional study. The pediatric heart transplant study. Circulation. 1997;96:227-231.

23. Gaies M, Cooper DS, Tabbutt S, et al. Collaborative quality improvement in the cardiac intensive care unit: development of the Paediatric Cardiac Critical Care Consortium (PC4). Cardiol Young. 2015;25:951-957.

24. Kipps AK, Cassidy SC, Strohacker CM, et al. Collective quality improvement in the paediatric cardiology acute care unit: establishment of the Pediatric Acute Care Cardiology Collaborative (PAC3). Cardiol Young. 2018;28:1019-1023.

25. Jenkins KJ, Beekman lii RH, Bergersen LJ, et al. Databases for assessing the outcomes of the treatment of patients with congenital and paediatric cardiac disease-the perspective of cardiology. Cardiol Young. 2008;18(Suppl 2):116-123.

26. Martin GR, Beekman RH, Ing FF, et al. The IMPACT registry: IMproving pediatric and adult congenital treatments. Semin Thorac Cardiovasc Surg Pediatr Card Surg Annu. 2010;13:20-25.

27. National Institutes of Health Grants Review Policy. Washington, DC: National Institutes of Health Policy on Scoring Procedures; 2018.

28. Harris PA, Taylor R, Thielke R, Payne J, Gonzalez N, Conde JG. Research electronic data capture (REDCap)-a metadata-driven methodology and workflow process for providing translational research informatics support. J Biomed Inform. 2009;42:377-381.

29. Mavroudis C, Bove EL, Cameron DE, et al. The Society of Thoracic Surgeons' National Congenital Heart Surgery Database. Ann Thorac Surg. 1995;59:554-556.

30. Petit CJ, Gao K, Goldstein BH, et al. Relation of aortic valve morphologic characteristics to aortic valve insufficiency and residual stenosis in children with congenital aortic stenosis undergoing balloon valvuloplasty. Am J Cardiol. 2016;117:972-979. 
31. Glatz AC, Petit CJ, Goldstein BH, et al. Comparison between patent ductus arteriosus stent and modified Blalock-Taussig shunt as palliation for infants with ductal-dependent pulmonary blood flow: insights from the congenital catheterization research collaborative. Circulation. 2018;137:589-601.

32. Petit CJ, Qureshi AM, Glatz AC, et al. Technical factors are associated with complications and repeat intervention in neonates undergoing transcatheter right ventricular decompression for pulmonary atresia and intact ventricular septum: results from the congenital catheterisation research collaborative. Cardiol Young. 2018;28:1042-1049.

33. Qureshi A, Goldstein B, Glatz A, et al. Classification scheme for ductal morphology in cyanotic patients with ductal-dependent pulmonary blood flow: influence of morphology on outcomes following ductal stenting. J Struct Heart Dis. 2017;2:A301.

34. Boucek D, Qureshi AM, Goldstein BH, Petit CJ, Glatz AC. BlalockTaussig shunt versus Patent ductus arteriosus stent as first palliation for ductal dependent pulmonary circulation lesions: a review of the literature. Congenit Heart Dis. In Press.

35. Aggarwal V, Petit CJ, Glatz AC, Goldstein BH, Qureshi AM. Stenting of the ductus arteriosus for ductal dependent pulmonary blood flow-current techniques and procedural considerations. Congenit Heart Dis. In Press.

36. Bauser-Heaton H, Goldstein B, Glatz A, et al. Percutaneous patent ductus arteriosus stenting using the carotid or axillary artery: a multicenter study from the Congenital Catheterization Research Collaborative. Catheter Cardiovasc Interv. 2018;91:V1-14.

37. Goldstein B, Petit C, Qureshi A, et al. Differences in cost of care by palliation strategy for infants with ductal-dependent pulmonary blood flow: results from the Congenital Catheterization Research Collaborative. Circulation. 2017;136:A17195.

38. Goldstein BH, Qureshi AM, Meadows J, et al. Impact of treatment strategy on outcomes in isolated pulmonary artery of ductal origin: a multicenter report from the Congenital Catheterization Research Collaborative. Catheter Cardiovasc Interv. 2018;91:V1-19.

39. Bauser-Heaton H, Qureshi A, Goldstein B, et al. Comparison of ductal arteriosus stent and Blalock-Taussig shunt as palliation for neonates with sole source ductal-dependent pulmonary blood flow: results from the Congenital Catheterization Research Collaborative. Circulation. 2018;138:A16867.

How to cite this article: Petit CJ, Qureshi AM, Glatz AC, et al. Comprehensive comparative outcomes in children with congenital heart disease: The rationale for the Congenital Catheterization Research Collaborative. Congenital Heart Disease. 2019;14:341-349. https://doi.org/10.1111/chd.12737 